\title{
Gas Chromatography-Mass Spectrometry Coupled with Multivariate Statistical Analysis to Identify the Alpha Glucosidase Inhibitors from Flesh of Salacca zalacca Fruits and Their Molecular Docking Studies
}

\author{
Mohammed S. M. Saleh $\left(\mathbb{D},{ }^{1,2}\right.$ Mohammad Jamshed Siddiqui $\mathbb{D}^{1},{ }^{1}$ Nabil Ali Al-Mekhlafi, ${ }^{3,4}$ \\ Hussah Abdullah Alshwyeh, ${ }^{5}$ Ahmed Mediani (i), ${ }^{6}$ Nor Hadiani Ismail, ${ }^{4}$ \\ and Yusof Kamisah ${ }^{2}$ \\ ${ }^{1}$ Department of Pharmaceutical Chemistry, Kulliyyah of Pharmacy, International Islamic University Malaysia, Indera Mahkota, \\ Kuantan, Pahang 25200, Malaysia \\ ${ }^{2}$ Department of Pharmacology, Faculty of Medicine, Universiti Kebangsaan Malaysia, Jalan Yaacob Latif, Cheras, \\ Kuala Lumpur 56000, Malaysia \\ ${ }^{3}$ Biochemical Technology Program, Department of Chemistry, Faculty of Applied Science, Dhamar University, P.O. Box 87246, \\ Dhamar, Yemen \\ ${ }^{4}$ Atta-ur-Rahman Institute for Natural Product Discovery, Universiti Teknologi MARA Selangor Branch, Puncak Alam Campus, \\ Bandar Puncak Alam, Kuala Selangor, Selangor 42300, Malaysia \\ ${ }^{5}$ Department of Biology, College of Science, Imam Abdulrahman Bin Faisal University (IAU), \\ Dammam 31441-1982, Saudi Arabia \\ ${ }^{6}$ Institute of Systems Biology (INBIOSIS), Universiti Kebangsaan Malaysia, UKM Bangi, Selangor 43600, Malaysia
}

Correspondence should be addressed to Mohammad Jamshed Siddiqui; jamshed_siddiqui@iium.edu.my and Ahmed Mediani; medianiahmed47@gmail.com

Received 17 September 2020; Revised 30 December 2020; Accepted 11 January 2021; Published 27 January 2021

Academic Editor: Francisco Solano

Copyright ( $(2021$ Mohammed S. M. Saleh et al. This is an open access article distributed under the Creative Commons Attribution License, which permits unrestricted use, distribution, and reproduction in any medium, provided the original work is properly cited.

\footnotetext{
Fruit of salak (Salaaca zalacca) is traditionally used and commercialized as an antidiabetic agent. However, scientific evidence to prove this folk claim is quite lacking. Therefore, this research was aimed to evaluate the $\alpha$-glucosidase inhibition activity of S. zalacca fruit and identify the bioactive compounds. The fruits were extracted by different ratios of ethanol and water $(0,20,40$, $60,80,100 \%, \mathrm{v} / \mathrm{v})$ to get E0 (100\% water), E20 (20\% ethanol), E40 (40\% ethanol), E60 (60\% ethanol), E80 ( $80 \%$ ethanol), and E100 (100\% ethanol) extracts. The extracts obtained were subjected to the $\alpha$-glucosidase inhibitory assay. Gas chromatography-mass spectrometry- (GC-MS-) based metabolomics approach was used in profiling the bioactive metabolites present in the extracts. Orthogonal partial least square (OPLS) was used to correlate GC-MS data and $\alpha$-glucosidase assay results to identify the possible chemical markers. All active compounds identified were subjected to molecular docking. The extracts from the $S$. zalacca fruit showed potent inhibition activity against $\alpha$-glucosidase. The $\mathrm{IC}_{50}$ values from the $\alpha$-glucosidase inhibitory assay ranged between 16 and $275 \mu \mathrm{g} / \mathrm{ml}$. Overall, E60 displayed significantly higher $\alpha$-glucosidase inhibition activity, while E0 showed the lowest $\alpha$-glucosidase inhibition activity. Major compounds detected in $S$. zalacca fruits were sugars, fatty acids, and sterols, including myo-inositol, palmitic acid, stearic acid, and $\beta$-sitosterol. Moreover, the results obtained from molecular docking indicated that palmitic acid and $\beta$-sitosterol were close to the active side of the enzyme. Some of the residues that interacted include HID295, ASN259, LEU313, LYS125, PHE159, VAL216, PHE178, TYR72, TYR158, HIE315, ARG315, and PHE303. The bioassay result strongly suggests that E60 extract from S. zalacca fruits has potential $\alpha$-glucosidase inhibitory activity. The hydrophobic compounds, including palmitic acid and $\beta$-sitosterol, were found to induce the $\alpha$-glucosidase inhibition activity.
} 


\section{Introduction}

Diabetes mellitus (DM) is a chronic metabolic disorder that may lead to other macro- and microvascular complications. The highest number of DM cases that have been reported to date was recorded in Asian countries, which cover about $60 \%$ of the world's DM patients [1]. World populations majorly practice conventional medicines relying on plantbased medicines due to the availability [2]. Various plants have been utilized as a traditional remedy for multiples diseases and conditions for centuries [3]. The effect and mechanism of most plants for the treatment of diabetes can be related to their inhibitory activities against $\alpha$-glucosidase enzyme. This intestinal enzyme is found in the brush border of the small intestine, responsible for catalysing the breakdown of polysaccharides into monosaccharides, thus reducing the postprandial glucose level, thereby decreasing the hyperglycaemia in DM patients [4]. In recent times, various plants and herbs have been extensively studied, providing scientific proof that the phytoconstituents present in plant matrix have various pharmacological effects on the human body system, such as fermented Chinese Ge-GenQin-Lian decoction and Radix Salvia Miltiorrhiza [5-7]. Salaaca zalacca is one example of a plant that is traditionally used as a hypoglycemic remedy, more commonly popular in Asian countries, including Malaysia, Myanmar, Indonesia, and Thailand $[8,9]$.

Salacca zalacca Gaertn Voss. is one of the palm species belonging to the family Arecaceae and is ubiquitously found all around Southeast Asia. S. zalacca fruits are a good source of vitamins, fibre, minerals, and carbohydrates [10]. Moreover, S. zalacca possesses antioxidant, anticancer, and antidiabetic effects [11-14], attributable to their high levels of vitamin C, Phenolics acids, and flavonoids [10]. In recent times, it has been claimed to possess antidiabetic activity $[12,14]$. Therefore, this study intends to initially analyse the $\alpha$-glucosidase (EC 3.2.1.20, $\alpha$-D-glucoside glucohydrolase) inhibitory activity of the $S$. zalacca fruit extracts. It is one of the pathways involved in the management of DM. One of the most appropriate methods to analyse these constituents are by using the metabolomics approach.

Typically, the analysis of metabolites can be achieved by herbal fingerprinting that requires the use of different chromatography and spectroscopy techniques, such as gas chromatography-mass spectrometry (GC-MS), and nuclear magnetic resonance (NMR) [15-18]. Therefore, the aim of the present study is to correlate the metabolic variation between $S$. zalacca extracts and $\alpha$-glucosidase inhibitory activity using the GC-MS-based metabolomics approach. This study is planned to identify the related bioactive compounds in different ratios of ethanol-water and molecular docking were also applied to predict the inhibitory activity.

\section{Materials and Methods}

2.1. Authentication of the Sample. Fresh S. zalacca (Gaertn.) Voss) fruits were harvested from a local farm in Bukit Sagu plantation, Felda Sagu, Kuantan, Pahang DM, Malaysia, during the month of May 2017. A voucher specimen has been submitted to the Kulliyyah of Pharmacy, International Islamic University of Malaysia. It was identified by Dr. Shamsul Khamis (Botanist) and deposited in the herbarium of Kulliyyah of Pharmacy with the voucher specimen number, i.e., PIIUM 0269-2 [VS-4].

2.2. Chemicals. Ethanol (analytical grade), dimethyl sulfoxide (DMSO) were obtained from Merck Germany (Darmstadt, Germany). $\alpha$-Glucosidase enzyme was obtained from Megazyme (Wicklow, Ireland). 4-nitrophenyl $\beta$-Dglucopyranoside substrate, methoxyamine hydrochloride, pyridine, $\mathrm{N}$-Methyl-N-(trimethylsilyl) trifluoroacetamide, and quercetin ( $\geq 95 \%$ HPLC) were obtained from SigmaAldrich (St. Louis, MO, USA).

2.3. Preparation of Extracts for Metabolomics Studies. The fruits of $S$. zalaaca were cleaned with tap water and then peeled into peel and flesh. Immediately, the separated fruits flesh was cut into small cups to remove the seeds. Initially, the fruits flesh was weighed and dried using a freeze dryer. The freeze-dried samples were ground into a fine powder using a laboratory blender and then kept at $-80^{\circ} \mathrm{C}$ prior to extraction. Five grams of dried powdered fruit was suspended in different ratios of ethanol and water $(0,20,40,60$, $80,100 \%, \mathrm{v} / \mathrm{v})$. Approximately $150 \mathrm{~mL}$ of the desired solvent was mixed with powdered and shaken before being transferred to sonicator at $25^{\circ} \mathrm{C}$ for $1 \mathrm{~h}$. A total of 36 extracts were obtained and filtered through a Whatman no. 1 filter paper. Finally, the filtered solutions were evaporated using a rotary evaporator at $40 \pm 1^{\circ} \mathrm{C}$. The stock concentrations of extracts were dried using a freeze dryer. The obtained amount of the extracts was weighed before keeping at $-80^{\circ} \mathrm{C}$ prior to further analysis [4].

2.4. $\alpha$-Glucosidase Inhibitory Activity. The $\alpha$-glucosidase inhibitory activity was measured using a method described by Collins et al. [19] with slight modifications. Different dilutions of the $S$. zalacca extracts were prepared in triplicate (i.e., $320,160,80,40,20,10$, and $5 \mu \mathrm{g} / \mathrm{mL}$ ). Then, $10 \mu \mathrm{L}$ of each dilution was added to $100 \mu \mathrm{L}$ of $30 \mathrm{mM}$ phosphate buffer $\mathrm{pH}$ 6.5. After that. $15 \mu \mathrm{L}$ of $\alpha$-glucosidase enzyme solution $(0.02 \mathrm{U} /$ well $)$ was added. The mixture was allowed to stand for $5 \mathrm{~min}$ before adding $75 \mu \mathrm{L}$ of the substrate (PNPG) (6 mg in $20 \mathrm{~mL}$ of $50 \mathrm{mM}$ phosphate buffer $\mathrm{pH} 6.5$ ). A total of $50 \mu \mathrm{L}$ of $(2 \mathrm{M})$ glycine of $\mathrm{pH} 10$ was added to the mixture to stop the reaction. The mixture was immediately measuring the absorbance at $405 \mathrm{~nm}$. The $\alpha$-glucosidase inhibition was decreased using the equation:

$$
\begin{aligned}
& \alpha \text { - glucosidase inhibitory activity (\%) } \\
& \quad=\left[\left(A_{\text {control }}-A_{\text {sample }}\right) / A_{\text {control }}\right] \times 100 \% .
\end{aligned}
$$

$\mathrm{IC}_{50}$ of the extract was determined from the graph of $\alpha$-glucosidase inhibitory activity (\%) against the amount of extract $(\mu \mathrm{g})$. 


\subsection{GC-MS Analysis}

2.5.1. Derivatization Procedure. The samples of extracts were derivatized using N-Methyl-N-(trimethylsilyl) trifluoroacetamide (MSTFA) and methoxyamine $\mathrm{HCl}$ for GCMS analysis following the method described by Robinson et al. (2005) [20]. Briefly, about $12.5 \mathrm{mg} \mathrm{S}$. zalacca fruits ethanolic extract was dissolved in $25 \mu \mathrm{L}$ pyridine in a $1 \mathrm{~mL}$ Eppendorf microcentrifuge tube. The mixture was initially vortexed for $2 \mathrm{~min}$ then further sonicated for $20 \mathrm{~min}$ at $30^{\circ} \mathrm{C}$. About $50 \mu \mathrm{L}(20 \mathrm{mg} / \mathrm{mL}$ in pyridine $)$ was added to the sample solution and incubated for $2 \mathrm{~h}$ at $60^{\circ} \mathrm{C}$. About $150 \mu \mathrm{L}$ of MSTFA was added and incubated again for $30 \mathrm{~min}$ at $60^{\circ} \mathrm{C}$. The derived sample was filtered using a microsyringe filter and equilibrated to room temperature overnight.

2.5.2. GC-MS-Based Metabolomics. GC-MS analysis of the S. zalacca fruits ethanolic extracts was carried out following the method described by Saleh et al. [11]. GC-MS analysis was performed on an Agilent 6890 gas chromatograph equipped with a 5973-mass selective detector using an electron impact ionization. A DB-5MS, 5\% phenyl methyl siloxane column with an inner diameter of $250 \mu \mathrm{m}$, and a film thickness of $250 \mu \mathrm{m}$ attached with an autosampler (Agilent, Santa Clara, United States) was used for the separation. A volume of $1 \mu \mathrm{L}$ sample was injected in splitless mode into GC-MS. The initial oven temperature was set at $180^{\circ} \mathrm{C}$ and the hold time $10 \mathrm{~min}$ and then increased to $220^{\circ} \mathrm{C}$ at a rate of $20^{\circ} \mathrm{C} / \mathrm{min}$ with the hold time of $5 \mathrm{~min}$. The final oven temperature was $315^{\circ} \mathrm{C}$ at a rate of $30^{\circ} \mathrm{C} / \mathrm{min}$ with a hold time of $10 \mathrm{~min}$, and the total run time was $30.16 \mathrm{~min}$. Helium gas was used as carrier gas at a flow rate of $1.5 \mathrm{~mL} /$ $\min$. The injector and detector were set to $330^{\circ} \mathrm{C}$ and $250^{\circ} \mathrm{C}$, respectively. Mass spectra were acquired using a full scan and a monitoring mode in a range of $50-550 \mathrm{~m} / z$ after a solvent delay of $6 \mathrm{~min}$. Identification of compounds was carried out by matching their peak spectra with the NIST14 library.

For GC-MS based metabolomics, raw chromatograms were converted into CDF format. This preprocessing of all the raw data into a matrix data includes systematic noise filtration, data binning, automatic peak detection, and chromatographic alignment. Baseline correction of the total ion chromatogram (TIC), integration, and peak picking were done using ACD/Spec Manager v.12.00 (Advanced Chemistry Development, Inc., ACD/Labs Toronto, Canada). Identification of metabolites was performed by matching their respective spectra with the NIST library. The XCMS package in $R$ version 2.15.1 was applied to align the GC-MS chromatograms using the following parameters; $\mathrm{xcms}$ set $(\mathrm{fwhm}=30$, step $=0.1$, method $=$ bin $)$ and group $(\mathrm{bw}=10)$. The information on $\alpha$-glucosidase activity was added to the converted excel format [16].

2.6. Statistical Analysis. The results were calculated as the mean \pm SD from six separate experiments $(n=6)$. The data were analyzed by one-way ANOVA test followed by Tukey's test using Minitab version 17. 0.

\section{Results}

3.1. Extraction Yield. Table 1 displays the trend of the extraction yield of the $S$. zalacca fruits extracts. The yield of extraction varied with the ratio of the water and ethanol of the solvents used, with the highest yield obtained from $80 \%$ hydroethanolic solvent (E80) as $75.5 \%$ and the least yield obtained from the ethanolic (100\%, E100) solvent as $20.2 \%$ only. The trend of this result can be simplified in the order as $80 \%(\mathrm{E} 80)>60 \%(\mathrm{E} 60)>0 \%(\mathrm{E} 0)>40 \%(\mathrm{E} 40)>20 \%(\mathrm{E} 20)$ $>100 \%(\mathrm{E} 100)$.

3.2. Inhibitory Activity of S. zalacca Fruits Extracts against $\alpha$-Glucosidase. The activity of S. zalacca extract on the $\alpha$-glucosidase enzyme is described in Table 1 . Results revealed that the highest $\alpha$-glucosidase inhibition activity was found in E60 extract ( IC $_{50} 16.48 \mu \mathrm{g} / \mathrm{mL}$ ), followed by E100 extract ( $\mathrm{IC}_{50} 20.08 \mu \mathrm{g} / \mathrm{mL}$ ), and followed by E80 ( $\mathrm{IC}_{50}$ $25.09 \mu \mathrm{g} / \mathrm{mL}$ ). On the other hand, the E0 extract had the lowest $\alpha$-glucosidase inhibition activity with $\mathrm{IC}_{50} 280$. $30 \mu \mathrm{g} / \mathrm{mL}$.

\subsection{Metabolite Profiling of S. zalacca Fruits Based on Metabolomics Approach}

3.3.1. GC-MS-Based Metabolites Profiling. When chemical profiling using GC-MS, derivatization is required for the metabolites to be converted into volatile compounds such as acetyl trimethyl silane (TMS) ethers or oxime-TMS ethers [21]. Flame ionization detectors or mass spectrometers are mostly used for detection. Confirmation of the identified metabolites was achieved by carefully examining the spectral pattern and comparison with the GC-MS NIST14 database library. The metabolites identified mostly belonged to sugars, sterols, as well as fatty acids that include D-(-)-fructofuranose, D-(-)-fructose, D-psicofuranose, D-glucose, sucrose, $\mathrm{D}-(+)$-trehalose, myo-inositol, $\beta$-D-glucopyranose, $\mathrm{D}-(+)-$ xylose, $\mathrm{D}$-(+)-talofuranose, $\alpha$-D-glucopyranose, D-psicopyranose, $\beta$-sitosterol, stearic acid, palmitic acid, and citric acid (Supplementary Figure S1).

All these metabolites have been labelled and indicated in each of the extracts' chromatogram analyzed. Specifically, three metabolites were found to be abundant in E60, E80, and E100 extracts, namely stearic acid and palmitic acid, $\beta$-sitosterol when compared to E0, E20, and E40. It is pertinent to note that there was a decrease in the intensity of the metabolites in the extract as the polarity of the solvent increased by increasing the amount of water in ethanol. In the meantime, sucrose and D-glucose were found to be dominant in the highly polar E0, E20, and E40 extracts. It is observable that a consecutive decrease in water ratio resulted in a decrease in the polarity. This results in metabolites with less polarity abundantly found in E60, E80, and E100. These include palmitic acid, stearic acid, and $\beta$-sitosterol which might be attributed to the $\alpha$-glucosidase inhibitory activity of $S$. zalacca fruits hydroethanolic extracts. 
TABLE 1: Extraction yield and $\alpha$-glucosidase inhibitory activity of $S$. zalacca fruit extracts.

\begin{tabular}{lcc}
\hline Extracts (ethanol/water) & Percentage yield $(\%)$ & $\alpha$-Glucosidase inhibitory activity IC $\mathrm{C}_{50}(\mu \mathrm{g} / \mathrm{mL})$ \\
\hline $0 \%(\mathrm{E} 0)$ & $69.4 \pm 3.6^{\mathrm{b}, \mathrm{c}}$ & $280.30 \pm 20.34^{\mathrm{a}}$ \\
$20 \%(\mathrm{E} 20)$ & $67.2 \pm 2.4^{\mathrm{c}}$ & $150.90 \pm 14.32^{\mathrm{b}}$ \\
$40 \%(\mathrm{E} 40)$ & $67.7 \pm 2.1^{\mathrm{c}}$ & $182 \pm 19.32^{\mathrm{c}}$ \\
$60 \%(\mathrm{E} 6)$ & $72.6 \pm 2.7^{\mathrm{a}, \mathrm{b}}$ & $16.48 \pm 1.20^{\mathrm{d}, \mathrm{e}}$ \\
$80 \%(\mathrm{E} 80)$ & $75.5 \pm 2.2^{\mathrm{a}}$ & $25.09 \pm 1.54^{\mathrm{d}}$ \\
$100 \%($ E100) & $20.2 \pm 1.4^{\mathrm{d}}$ & $20.08 \pm 1.24^{\mathrm{d}, \mathrm{e}}$ \\
Quercetin & nd & $3.54 \pm 0.32^{\mathrm{e}}$ \\
\hline
\end{tabular}

Values represent the mean \pm standard deviation $(n=6)$. The values that do not share a letter are significantly different at $P<0.05$. nd $=$ not determined.

3.3.2. Multivariate Data Analysis. One of the ways of identifying bioactive metabolites is through the GC-MSbased metabolomics approach using MVDA to develop an orthogonal partial least-squares (OPLS) model. Two results are usually automatically generated, namely OPLS components 1 and 2 . The $\mathrm{m} / z$ (mass-to-charge ratio) of certain retention time was generated along with $1 / \mathrm{IC}_{50}(\mu \mathrm{g} / \mathrm{mL})$ values of the analytes that were taken as variables $x$ and $y$, respectively. The major aim of performing OPLS is to determine differences in the constituents among the analytes and to identify the $\mathrm{m} / z$ values responsible for the pharmacological activity.

The OPLS model can be assessed through different parameters, namely, the permutation test, the goodness of fit, and the capacity of this model in predicting the $y$-value via the predicted plot. Meanwhile, the cumulative values $R^{2} Y$ explains more on goodness of fit which indicates the variation percentage of the response as explained by the model, whereas the $Q^{2} Y$, the cumulative value, represents variation percentage of response based on model prediction in accordance with the cross validation. If the cumulative values of $R^{2} \mathrm{Y}$ and $\mathrm{Q}^{2} \mathrm{Y}$ are observed greater than 0.5 , then the fitness of the model and predictive ability are considered satisfactory [20]. The $\mathrm{R}^{2} \mathrm{Y}$ and $\mathrm{Q}^{2} \mathrm{Y}$ values obtained in this study were 0.856 and 0.925 , respectively. In addition to the above-mentioned parameters, other ones that were also considered significant in determining the model accuracy and performance are the root-mean-square error of crossvalidation $\left(\mathrm{RMSE}_{\mathrm{CV}}\right)$ and root-mean-square errors of estimation $\left(\mathrm{RMSE}_{\mathrm{E}}\right) \cdot \mathrm{RMSE}_{\mathrm{CV}}$ simply measures the quality of the model in terms of new sample prediction. Low $\mathrm{RMSE}_{\mathrm{CV}}$ values indicate higher predictive accuracy of the sample. $\mathrm{RMSE}_{\mathrm{E}}$, on the other hand, measures the average deviation of the model from the data.

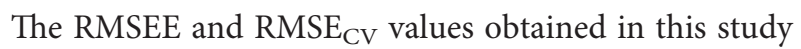
were 0.356078 and 0.376476 , respectively. The score scatter plot of the OPLS model is shown in Figure 1(a). It indicates good separation of the samples in terms of the chemical profile and pharmacological activity $\left(\mathrm{IC}_{50}\right.$ value) of the samples. The positive OPLS component 1 consists of samples with higher activity (E60, E100, and E80) having $\mathrm{IC}_{50}$ value less than $30 \mu \mathrm{g} / \mathrm{mL}$, whereas the less active samples (E40, E20, and E0) were found at the negative OPLS component 1.

The loading plot of different extracts of S. zalacca fruits hydroethanolic extracts is shown in Figure 1(b). The metabolites in each sample are represented by each peak. The compounds identification and details are shown in Table 2.
Peaks labelled as $1,2,3,5,6,7,9,11,13,15$, and 16 represent D-psicofuranose, D-(-)-fructofuranose, D-(-)-fructose, sucrose, D-glucose, D-(+)-trehalose, citric acid, D-(+)-talofuranose, $\beta$-D-glucopyranose, $\alpha$-D-glucopyranose, D- $(+)$ xylose, and D-psicopyranose, respectively. They are observed opposite to the $1 / \mathrm{IC}_{50}$ value. These compounds are assumed to decrease $\alpha$-glucosidase inhibitory activity. They were found to be abundant in sample, E0, E20, and E40, with the $\mathrm{IC}_{50}$ value greater than $100 \mu \mathrm{g} / \mathrm{mL}$, which indicate that these compounds possess less activity. The remaining peaks found in the same position aligned with the $\mathrm{IC}_{50}$ value were efficient in inhibiting $\alpha$-glucosidase activity. These metabolites were identified as plant fatty acids, carbocyclic sugar, and sterol, such as palmitic acid, stearic acid, myo-inositol, and $\beta$-sitosterol.

The permutation test plot shown in Figure 2 describes the validation of the developed OPLS model. The plot displayed the fraction intercepts of the sum of the squares intercept $\mathrm{Y}$-value and the predictive ability of the model intercept $\mathrm{Y}$-value. In this study, the $\mathrm{R}^{2} \mathrm{Y}$ and $\mathrm{Q}^{2} \mathrm{Y}$ values obtained were 0.507 and -0.408 , respectively. These values are acceptable because $R^{2}$ and $Q^{2}$ are less than or equal to -0.5 and 0.5 , respectively. Meanwhile, the plot showing the observed versus predicted plot predicts the OPLS model accuracy along its validation using $R^{2}$ value obtained from the regression. This plot is valid if the $R^{2}$ value is greater than 0.9 . Therefore, the result obtained is valid with the $R^{2}$ value of 0.9455 .

3.4. Docking of Bioactive Compounds. All identified compounds responsible for the $\alpha$-glucosidase inhibition activity were docked to the Saccharomyces cerevisiae isomaltase (SCI) crystal structure (PDB ID: 3A4A). The conformations showing the lowest binding energy for the compounds along with the interacting residues are summarised in Table 3. Based on the AutoDock 1.5.6 simulation result displayed in Table 3 , the inhibitor complex of $\alpha$-glucosidase-quercetin, which was used as positive control showed $-8.25 \mathrm{kcal} / \mathrm{mol}$ binding energy having six hydrogen bonds with the interacting residues LYS156, ASN415, GLU411, THR310, LEU313, and PRO312 along with hydrophobic interactions involving ARG315 and PHE314. The stearic acid binding affinity in complex with $\alpha$-glucosidase was observed to be $-3.80 \mathrm{kcal} / \mathrm{mol}$. One $\mathrm{H}$-bond was displayed in the complex including CYS342 with hydrophobic interactions with LYS16 and AGR263. Meanwhile, for 


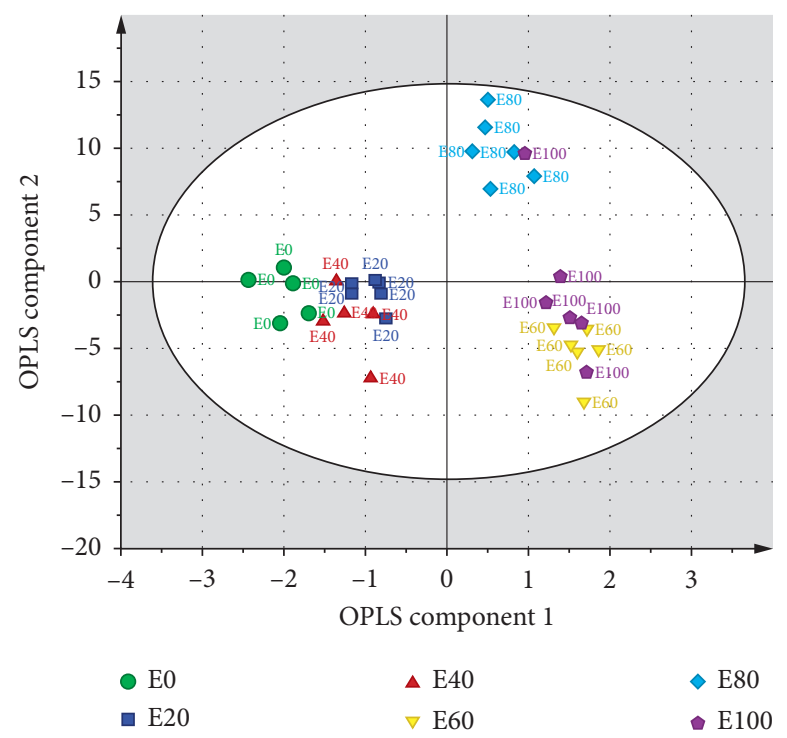

(a)

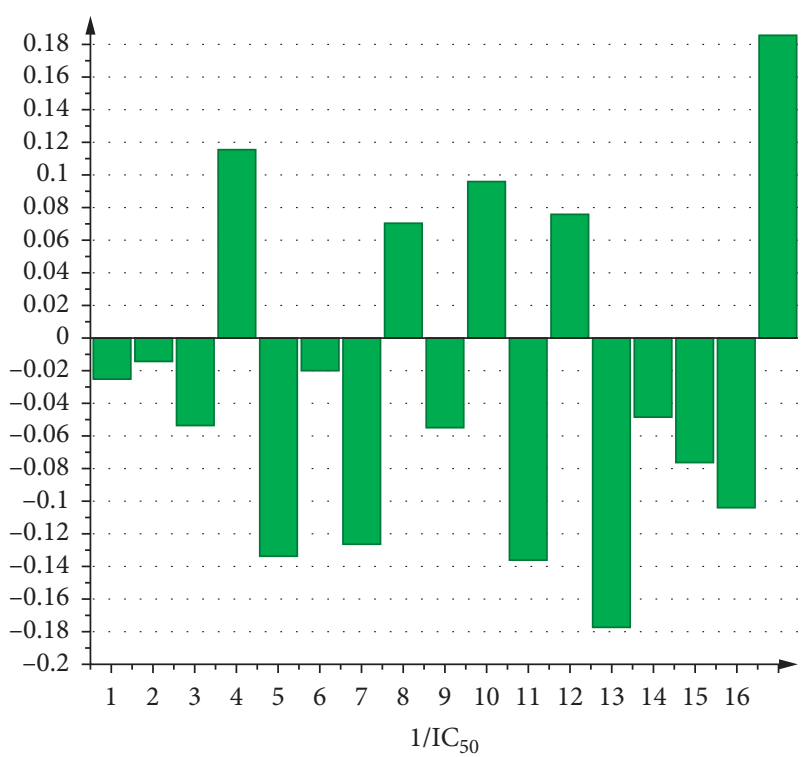

(b)

Figure 1: (a) OPLS score scatter plot of different extracts of $S$. zalacca fruits; (b) the PC1 loading column plot of different extracts of S. zalacca fruits.

TABLe 2: The metabolites identified in the S. zalacca fruits hydroethanolic extracts using GC-MS-based metabolomics.

\begin{tabular}{|c|c|c|c|c|c|}
\hline No. & RT (min) & Similarity index (\%) & Molecular weight & Molecular formula & Tentative metabolites \\
\hline 1 & 20.032 & 90 & 180.16 & $\mathrm{C}_{6} \mathrm{H}_{12} \mathrm{O}_{6}$ & D-psicofuranose \\
\hline 2 & 20.638 & 87 & 180.16 & $\mathrm{C}_{6} \mathrm{H}_{12} \mathrm{O}_{6}$ & D-(-)-fructofuranose \\
\hline 9 & 21.175 & 91 & 192.17 & $\mathrm{C}_{6} \mathrm{H}_{8} \mathrm{O}_{7}$ & Citric acid \\
\hline 16 & 23.215 & 81 & 180.16 & $\mathrm{C}_{6} \mathrm{H}_{12} \mathrm{O}_{6}$ & D-psicopyranose \\
\hline 11 & 23.387 & 94 & 180.16 & $\mathrm{C}_{6} \mathrm{H}_{12} \mathrm{O}_{6}$ & D-(+)-talofuranose \\
\hline 3 & 25.719 & 91 & 180.16 & $\mathrm{C}_{6} \mathrm{H}_{12} \mathrm{O}_{6}$ & D-(-)-fructose \\
\hline 13 & 26.89 & 95 & 180.16 & $\mathrm{C}_{6} \mathrm{H}_{12} \mathrm{O}_{6}$ & $\alpha$-D-glucopyranose \\
\hline 6 & 27.056 & 90 & 180.16 & $\mathrm{C}_{6} \mathrm{H}_{12} \mathrm{O}_{6}$ & D-glucose \\
\hline 14 & 28.685 & 93 & 180.16 & $\mathrm{C}_{6} \mathrm{H}_{12} \mathrm{O}_{6}$ & $\beta$-D-glucopyranose \\
\hline 10 & 29.331 & 99 & 256.42 & $\mathrm{C}_{16} \mathrm{H}_{32} \mathrm{O}_{2}$ & Palmitic acid \\
\hline 15 & 25.742 & 91 & 150.13 & $\mathrm{C}_{5} \mathrm{H}_{10} \mathrm{O}_{5}$ & D-(+)-xylose \\
\hline 8 & 29.685 & 93 & 180.16 & $\mathrm{C}_{6} \mathrm{H}_{12} \mathrm{O}_{6}$ & Myo-inositol \\
\hline 4 & 30.657 & 99 & 284.48 & $\mathrm{C}_{18} \mathrm{H}_{36} \mathrm{O}_{2}$ & Stearic acid \\
\hline 7 & 32.817 & 95 & 342.30 & $\mathrm{C}_{12} \mathrm{H}_{22} \mathrm{O}_{11}$ & $\mathrm{D}$-(+)-trehalose \\
\hline 12 & 37.195 & 99 & 414.71 & $\mathrm{C}_{29} \mathrm{H}_{50} \mathrm{O}$ & $\beta$-Sitosterol \\
\hline 5 & 37.601 & 91 & 342.30 & $\mathrm{C}_{12} \mathrm{H}_{22} \mathrm{O}_{11}$ & Sucrose \\
\hline
\end{tabular}

$\alpha$-glucosidase- myo-inositol complex, the binding affinity value of $-5.68 \mathrm{kcal} / \mathrm{mol}$ was observed. Three residues including PRO456, AGR467, and LYS406 were found to interact with myo-inositol through $\mathrm{H}$-bonds, whereas TYR470 was found to interact via $\pi$-bond. It is interesting to know that $\beta$-sitosterol, despite interacting with just two residues by $\mathrm{H}$-bonding with its $\mathrm{O}-\mathrm{H}$ group, LEU313, and LYS125, it comparably displayed better binding energy of $-9.55 \mathrm{kcal} / \mathrm{mol}$ than the quercetin (positive control). The hydrophobic contacts appeared to dominate in $\alpha$-glucosidase- $\beta$-sitosterol complex, perhaps as a result of its alkyl group and cyclic skeleton that is preferably bound to PHE159, VAL216, PHE178, TYR72, TYR158, HIE315, ARG315, and PHE303.
The crystallized ligand, $\alpha$-D-glucose (ADG) demonstrated lower binding energy of $6.02 \mathrm{kcal} / \mathrm{mol}$ as compared to $\beta$-sitosterol and quercetin (positive control). However, the $\mathrm{H}$-bond existing between $\mathrm{ADG}$ and the protein showed a stable interaction with nine amino acid residues, including ASH69, HIE112, ARG213, ASH215, GLH277, HIE351, ASP352, and ARG442 having hydrophobic interaction with TYR72. Unlike the docked ADG, results obtained from docking indicated that the binding of tested compounds was mostly supported through hydrophobic contacts with $\alpha$-glucosidase. Our result corroborates with those reported by Seong et al. [21] in which the isoflavones, i.e., daidzein, genistein, and calycosin, exhibited more hydrophobic contacts with comparable residues, such as ILE262, ARG263, 


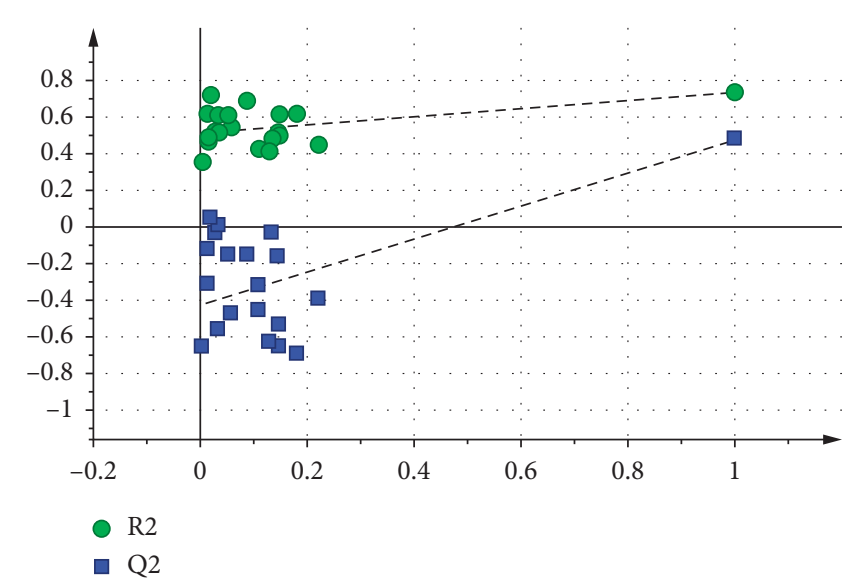

Figure 2: Permutation test of different extracts of S. zalacca fruits.

TABle 3: Molecular interaction results of $\alpha$-glucosidase enzyme protein with tentative compounds identified using GC-MS-based metabolomics.

\begin{tabular}{lccc}
\hline Compound control & $\begin{array}{c}\text { Binding energy } \\
(\mathrm{kcal} / \mathrm{mol})\end{array}$ & H-bond interacting residues & Other interacting residues \\
\hline $\begin{array}{l}\alpha \text {-D-glucose (control } \\
\text { ligand) }\end{array}$ & -6.02 & ARG442, ASH69, HIE112, ASH215, GLH 277, & TYR72 \\
$\begin{array}{l}\text { Quercetin (positive } \\
\text { control) }\end{array}$ & -8.24 & LYS156, ASN415, GLU411, THR310, LEU313, & ARG315, PHE314 \\
Stearic acid & -3.80 & PRO312 & LYS16, AGR263 \\
Myo-inositol & -5.68 & CYS342 & TYR470 \\
Palmitic acid & -3.59 & PRO456, ARG467, LYS406 & - \\
$\beta$-Sitosterol & -9.55 & HID295, ASN259 & PHE159, VAL216, PHE178, TYR72, TYR158, \\
\hline
\end{tabular}

ILE272, and VAL266. The 2D diagram of the interaction between the protein residues and ADG, positive control, stearic acid, palmitic acid, myo-inositol, and $\beta$-sitosterol is shown in Figure 3. Moreover, the 3D superimposed diagram of the compounds and ADG is shown in Figure 4(a). It was also observed that the binding site for palmitic acid and $\beta$-sitosterol was close to the active site pocket (Figures 4 (b) and $4(\mathrm{c}))$.

\section{Discussion}

The $\alpha$-glucosidase enzyme is one of the key enzymes involved in the digestion of carbohydrates in humans. Its main function is to hydrolyse the carbohydrate to release glucose, which may lead to an increase in postprandial blood glucose level. Inhibition of this enzyme is one of the effective ways of controlling the postprandial blood glucose level, which is considered beneficial for diabetic patients. There are several synthetic drugs available in the market for the management and treatment of diabetes, such as migitol, acarbose, and vogibose. However, they are usually accompanied by serious side effects such as hepatic disorders and other negative gastrointestinal symptoms [22]. Therefore, plant-derived $\alpha$-glucosidase inhibitors are envisaged as preferable and safer as a means of management and prevention of diabetes. Metabolites obtained from plants have provided a wide range of pharmacological benefits to human health [23]. In many bioactivity analyses of plants, in vitro techniques are applied to determine the pharmacological effectiveness of plant metabolites. In determining the in vitro antidiabetic activity, various procedures are employed. The most important of it is the $\alpha$-glucosidase inhibitory assay. The pharmacological activity of plant metabolites is dependent on the type and numbers of functional groups present, the chemical structure, as well as the nature of substitution on the ring [24]. Currently, research on the use of medicinal plants has become a continuous effort due to the fact that numerous numbers of bioactive metabolites are distributed in plants with various pharmacological benefits. Water, a mixture of water and organic solvent and different organic solvents are employed to extract the potential metabolites from plants through different methods $[4,11]$. The polarity of the extracting solvent determines the type of metabolite and hence the bioactivities found in the extract. Due to this fact, different solvents were used in this study to obtain S. zalacca fruits extracts so as to determine different metabolites having $\alpha$-glucosidase inhibition potential.

The results obtained showed a decrease in the ratio of water, with a moderate decrease in polarity, suggesting that the metabolites responsible for the inhibitory activity are the less polar ones such as sterols and fatty acids. This outcome signifies that the extracting solvent has a greater influence on 


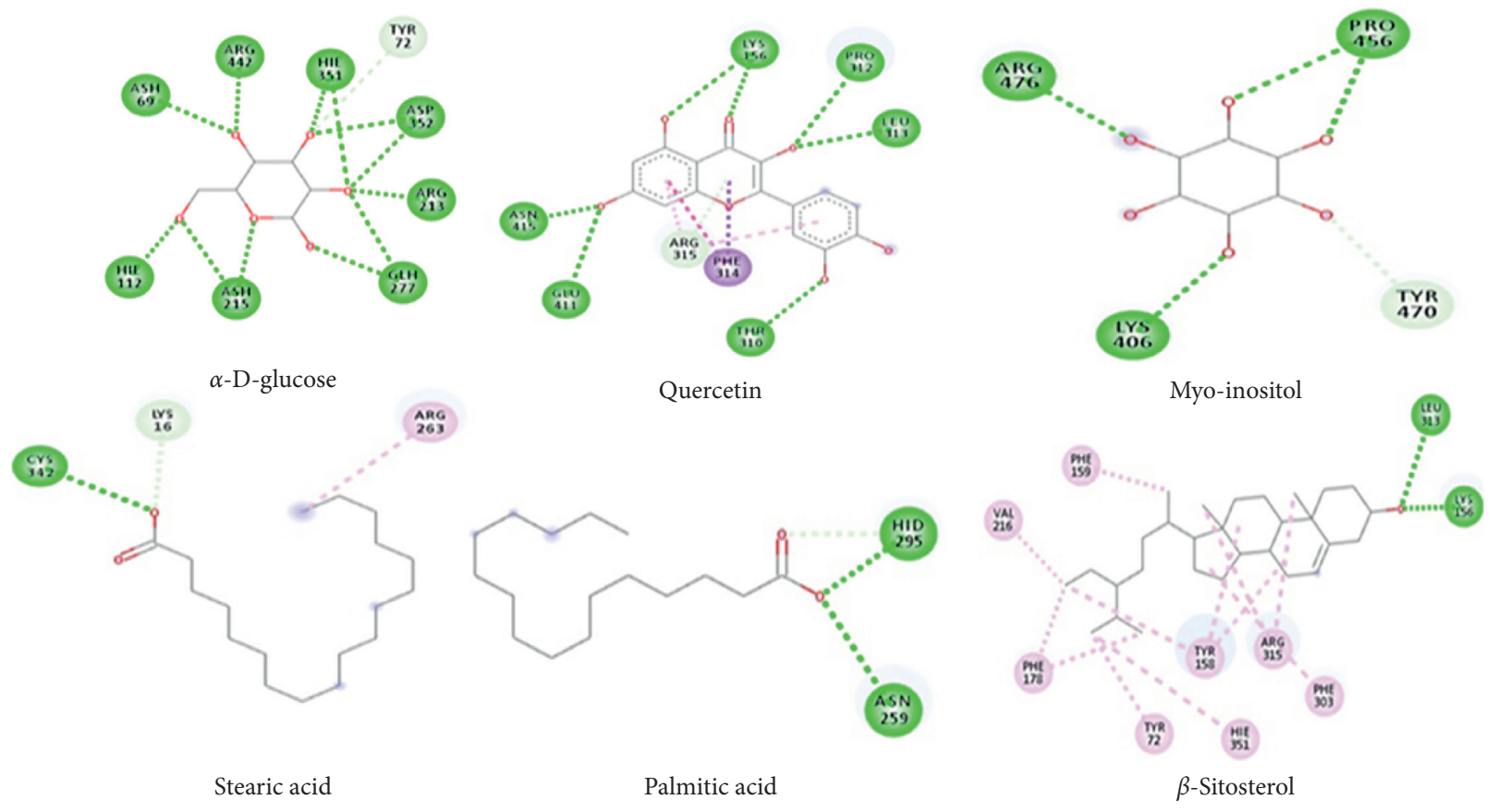

Figure 3: The 2D structure of protein-ligand conformations of PDB3A4A and ligands.

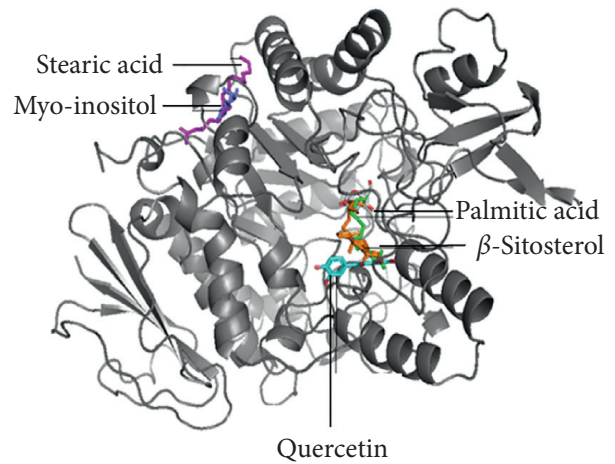

(a)

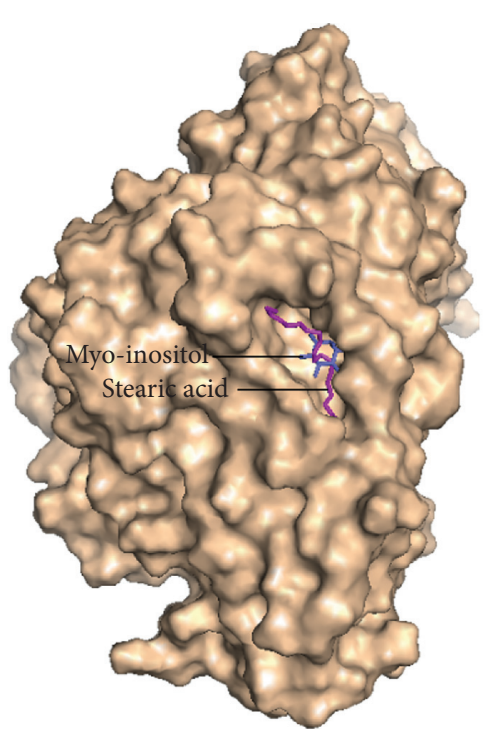

(b)

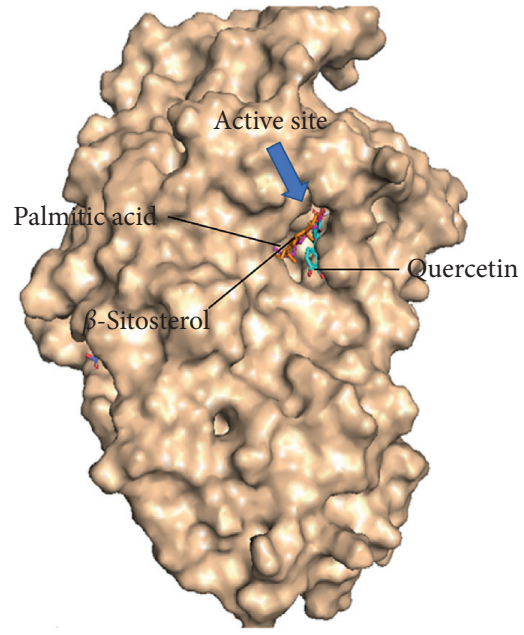

(c)

FIGURE 4: (a) Superimposition of the identified compounds, quercetin, and ADG in the binding site of enzyme; (b) and (c) predicted binding mode of the identified compounds and quercetin with $\alpha$-glucosidase on the molecular surface.

the bioactivity of the extract. Some of the compounds identified have previously been reported in the literature to exhibit in vitro $\alpha$-glucosidase inhibitory effect. The investigation conducted on $\alpha$-glucosidase inhibitory activity of palmitic acid and other fatty acids by Liu et al. [25] showed that palmitic acid exhibited inhibitory activity with the $\mathrm{IC}_{50}$ value $>400 \mu \mathrm{g} / \mathrm{mL}$ which was in contradiction with the value earlier reported in other studies for the same compound, i.e., IC $_{50} 11.53 \mu \mathrm{g} / \mathrm{mL}$ and $24.09 \mu \mathrm{g} / \mathrm{mL}[26,27]$. Aside palmitic acid, other compound that was found active against $\alpha$-glucosidase was stearic acid $5.3 \%$ inhibition at $10 \mu \mathrm{g} / \mathrm{mL}$ [28].

In another study, myo-inositol and $\beta$-sitosterol with other compounds were purified from the methanol extract of Tournefortia hartwegiana (Boraginaceae) (METh). An oral glucose tolerance test (OGTT) was conducted using a different substrate such as glucose, maltose, and sucrose through intragastric administration. Results obtained 
showed that the increased plasma glucose level was significantly $(P<0.05)$ suppressed by the extract containing myoinositol and $\beta$-sitosterol after the administration of the substrates. Moreover, METh also exhibited an in vitro $\alpha$-glucosidase inhibitory activity in a concentration-dependent manner $\left(\mathrm{IC}_{50}: 3.16 \mathrm{mg} / \mathrm{mL}\right)$. Another investigation was conducted by Kim et al. [29] on myo-inositol to determine its effect on the differentiation, lipolysis of adipocytes, insulin-stimulated glucose uptake and to test whether the antidiabetic activity of myo-inositol via is mediated through adipocytes. In the study, 3T3-L1 cells were treated with varying concentrations of myo-inositol. Lipid accumulation was increased after exposure to myo-inositol dosedependently $(P<0.01)$. An increase in adipogenesis was observed along with insulin-stimulated glucose uptake in adipocytes through activation of insulin signalling. Furthermore, lipolysis in matured adipocytes was also inhibited. Therefore, myo-inositol possesses a promising therapeutic property for improving insulin sensitivities in adipocytes through the increase in lipid storage capacity, glucose uptake, and inhibition of free fatty acid formation.

Chemical and structural derivatives of plant sterols are phytochemicals that are found ubiquitously in many plant parts. Many of them have been previously identified from plant extracts; these include $\beta$-sitosterol. Research conducted on the antidiabetic and antioxidant potentials of $\beta$-sitosterol in STZ-induced rats showed that there was a significant decrease in glycated haemoglobin, nitric oxide, and serum glucose and with a simultaneous increase in serum insulin levels. In addition to that, pancreatic antioxidants levels were also increased with a drastic reduction in thiobarbituric acid-reactive substances [30]. In another study, $\beta$-sitosterol, which is an active phytosterol in the S. surattense stem bark, has also been reported to be useful in the treatment of coronary artery disease, breast cancer, prostate cancer, and hypercholesterolemia [31]. Apoptosis induced by caspase 8 activity, FAS receptor levels, and phosphorylation of extracellular signal-regulated kinase and mitogen-activated protein kinase has been suggested to be responsible for the chemopreventive property of $\beta$-sitosterol with no cytotoxicity to normal cells $[32,33]$.

Although from the structure-activity relationship studies, the presence of multiple hydroxyl groups in a compound has been reported to be responsible for the $\alpha$-glucosidase inhibitory effect, yet, compounds identified using GC-MS in this study were mostly nonpolar compounds which include sterol, fatty acids, excluding myo-inositol. These compounds containing a few numbers of hydroxyl groups except myoinositol. Despite the same fact, these compounds were found to exhibit a certain level of $\alpha$-glucosidase inhibitory activity based on the literature. This could be as a result of interactions existing between protein and the inhibitors, thereby forming probable conformational complexes that may result in positive enzyme inhibitory effect of the identified inhibitors; myo-inositol, palmitic acid and stearic acid, and $\beta$-sitosterol.

With these results, it is deduced that the hydrophobic and $\pi$-interactions existing between the cyclic part of the compounds structure with hydrophobic side chains (TYR,
VAL, and PHE) of the enzyme could be responsible for the much higher activities of these nonpolar compounds, which is agreeable to the finding reported by Nussinov [34] and $\mathrm{Du}$ et al. [35]. In addition, the strong hydrogen bond also occurs between the compounds' hydroxyl group with the residues having both the polar (GLN and SER) and electrically charged side chains (ARG, ASP, and HIE (protonated HIS). This has been observed in quercetin and other similar compounds. Interaction between the residues and that of ADG was observed as shown through its binding energy. As illustrated in Table 3, there was an interaction between ADG and $\mathrm{ASH} 215$ residue that acted as a catalytic nucleophile. Furthermore, a strong $\mathrm{H}$-bond was also formed with GLU277 protonated GLU residue, which served as the general acid-base catalyst. Apart from this, ADG also showed interaction with ASP352, which stabilises the substrate during the catalytic reaction so as to strengthen the acid-base hydrolysis reaction [34, 36]. The $3 \mathrm{D}$ diagram in Figure 4(a) also indicated that the protein might have more than one site for ligand binding. This could induce allosteric control for catalytic reaction, which may cause changes in the active site, thereby resulting in the enzyme inhibition. Allosteric inhibitors are capable of modifying protein activity [35, 37]. In general view, the conjugated behaviour of a compound in which the conformational perturbations produced at one region of a macromolecule affects the active site and consequently, the enzyme activity confers the allosteric binding ability. In some occasions where the allosteric interaction is unstable, the binding residues stabilises it. This will eventually result in an altered functional site due to the equilibrium shifting toward this conformer or the allosteric ligand, thus causing the inhibition activity [38, 39]. Additionally, Figure 4(a) (the superimposed 3D structure) also suggested that the identified inhibitors using GC-MS displayed noncompetitive inhibition mode when compared to the control ligand, ADG, the known competitor. For instance, $\beta$-sitosterol, previously reported as a potential inhibitor binds away from the active site indicating the noncompetitive and allosteric binding mode. The binding of $\beta$-sitosterol was assisted through hydrophobic interactions that contributed to its binding energy, hence presumably responsible for its inhibitory activity. In this phenomenon, the conformational changes will be observed in the active site of the enzyme and therefore slows down the enzyme activity [39-41].

The predicted binding area in myo-inositol could be capable of inhibiting protein activity via a noncompetitive mode and those compounds with similar nature. Hydrophobic and $\pi$-interactions play a crucial role in the enzyme inhibitory activity of compounds with a lack of hydroxyl groups. The control ligand's binding site on $\alpha$-glucosidase within the active site, whereas the compounds such as quercetin and palmitic acid were observed to occupy the entrance of the pocket and not directly interacting with the residues within the active site. This suggests the existence of an allosteric binding site, which could probably cause alterations in the enzyme activity. Cleft closure may be induced in this arrangement to avoid the entrance of substrate, 
which then acts as noncompetitive inhibitors. In conclusion, molecular docking conducted in this study has enabled the prediction and provided supportive data for $\alpha$-glucosidase inhibitory activity of the bioactive compounds.

\section{Conclusion}

The study showed that E60 S. zalacca fruit revealed potent $\alpha$-glucosidase inhibitory activity. By GC-MS analysis, many compounds were detected in S. zalacca fruits, and the molecular docking results showed that palmitic acid and $\beta$-sitosterol were close to the active side of the enzyme. This study has provided the appropriate information on the potential antidiabetic action of S. zalacca fruits via $\alpha$-glucosidase inhibitory effect and the phytochemical constituents that may be responsible for this activity. This finding can be applied for further investigations of the S. zalacca fruits' beneficial uses and the development of the medicinal preparations for the management of diabetes.

\section{Data Availability}

The data used to support the findings of this study are included within the article and the supplementary information files. Any other data can be made available upon request.

\section{Conflicts of Interest}

The authors declare that they have no conflicts of interest.

\section{Acknowledgments}

The authors would like to acknowledge the support of Kulliyyah of Pharmacy, International Islamic University Malaysia.

\section{Supplementary Materials}

Figure S1: TIC of S. zalacca extracts. (Supplementary Materials)

\section{References}

[1] A. Ramachandran, C. Snehalatha, A. S. Shetty, and A. Nanditha, "Trends in prevalence of diabetes in Asian countries," World Journal of Diabetes, vol. 3, no. 6, pp. 110-117, 2012.

[2] M. S. M. Saleh and Y. Kamisah, "Potential medicinal plants for the treatment of dengue fever and severe acute respiratory syndrome-coronavirus," Biomolecules, vol. 11, no. 42, pp. 1$25,2021$.

[3] N. El Omari, K. Sayah, S. Fettach et al., "Evaluation of in vitro antioxidant and antidiabetic activities of Aristolochia longa extracts," Evidence-based Complementary and Alternative Medicine, vol. 2019, Article ID 7384735, 9 pages, 2019.

[4] M. S. M. Saleh, M. J. Siddiqui, S. Z. M. So'ad et al., "Correlation of FT-IR fingerprint and $\alpha$-glucosidase inhibitory activity of salak (Salacca zalacca) fruit extracts utilizing orthogonal partial least square," Molecules, vol. 23, no. 1434, 2018.

[5] Y. Yan, C. Du, Z. Li et al., "Comparing the antidiabetic effects and chemical profiles of raw and fermented Chinese Ge-Gen-
Qin-Lian decoction by integrating untargeted metabolomics and targeted analysis," Chinese Medicine (United Kingdom), vol. 13, no. 1, pp. 1-18, 2018.

[6] Y. Y. Yang, Z. Y. Wu, F. B. Xia et al., "Characterization of thrombin/factor Xa inhibitors in Rhizoma Chuanxiong through UPLC-MS-based multivariate statistical analysis," Chinese Medicine (United Kingdom), vol. 15, no. 1, pp. 1-14, 2020.

[7] Y. Y. Yang, Z. Y. Wu, H. Zhang et al., "LC-MS-based multivariate statistical analysis for the screening of potential thrombin/factor Xa inhibitors from Radix Salvia Miltiorrhiza," Chinese Medicine (United Kingdom), vol. 15, no. 1, pp. 1-13, 2020.

[8] E. Zubaidah, R. A. Ifadah, U. Kalsum et al., "Anti-diabetes activity of Kombucha prepared from different snake fruit cultivars," Nutrition \& Food Science, vol. 49, 2018.

[9] S. W. Qader, M. A. Abdulla, L. S. Chua, N. Najim, M. M. Zain, and S. Hamdan, "Antioxidant, total phenolic content and cytotoxicity evaluation of selected Malaysian plants," Molecules, vol. 16, no. 4, pp. 3433-3443, 2011.

[10] M. S. M. Saleh, M. J. Siddiqui, A. Mediani et al., "Salacca zalacca: a short review of the palm botany, pharmacological uses and phytochemistry," Asian Pacific Journal of Tropical Medicine, vol. 11, no. 12, pp. 645-652, 2018.

[11] M. S. M. Saleh, D. A. M. Bukhari, M. J. A. Siddiqui et al., "GCMS analysis of metabolites from soxhlet extraction, ultrasound-assisted extraction and supercritical fluid extraction of Salacca zalacca flesh and its alpha-glucosidase inhibitory activity," Natural Product Research, vol. 34, pp. 1-4, 2019.

[12] M. S. M. Saleh, M. J. Siddiqui, A. Mediani et al., "Modulation of metabolic alterations of obese diabetic rats upon treatment with Salacca zalacca fruits extract using ${ }^{1} \mathrm{H}$ NMR-based metabolomics," Food Research International, vol. 137, Article ID 109547, 2020.

[13] M. S. M Saleh, M. J. Siddiqui, S. Z. M. So’ad, S. Murugesu, A. Khatib, and M. M. Rahman, "Antioxidant and $\alpha$-glucosidase inhibitory activities and gas chromatography- mass spectrometry profile of salak (Salacca zalacca) fruit peel extracts," Pharmacognosy Research, vol. 10, pp. 24-30, 2018.

[14] S. P. Ong and C. L. Law, "Drying kinetics and antioxidant phytochemicals retention of salak fruit under different drying and pretreatment conditions," Drying Technology, vol. 29, no. 4, pp. 429-441, 2011.

[15] A. Nokhala, Q. U. Ahmed, M. S. M. Saleh, T. S. Nipun, A. a. Y. F. Khan, and M. J. Siddiqui, "Characterization of $\alpha$-glucosidase inhibitory activity of Tetracera scandens leaves by Fourier transform infrared spectroscopy-based metabolomics," Advances in Traditional Medicine, vol. 20, no. 2, pp. 169-180, 2020.

[16] S. Murugesu, V. Perumal, T. Balan et al., "The investigation of antioxidant and antidiabetic activities of Christia vespertilionis leaves extracts," South African Journal of Botany, vol. 133, pp. 227-235, 2020.

[17] M. Chandradevan, S. Simoh, A. Mediani, I. S. Ismail, and F. Abas, "1 H NMR-based metabolomics approach in investigating the chemical profile, antioxidant and anti-inflammatory activities of Gynura procumbens and Cleome gynandra," Plant Foods for Human Nutrition, vol. 75, 2020.

[18] N. Javadi, F. Abas, A. Mediani et al., "Effect of storage time on metabolite profile and alpha-glucosidase inhibitory activity of Cosmos caudatus leaves-GCMS based metabolomics approach," Journal of Food and Drug Analysis, vol. 23, no. 3, pp. 433-441, 2015. 
[19] R. A. Collins, T. B. Ng, W. P. Fong, C. C. Wan, and H. W. Yeung, "Inhibition of glycohydrolase enzymes by aqueous extracts of Chinese medicinal herbs in a microplate format," IUBMB Life, vol. 42, no. 6, pp. 1163-1169, 1997.

[20] A. R. Robinson, R. Gheneim, R. A. Kozak, D. D. Ellis, and S. D. Mansfield, "The potential of metabolite profiling as a selection tool for genotype discrimination in Populus," Journal of Experimental Botany, vol. 56, no. 421, pp. 28072819, 2005.

[21] J. W. Allwood, D. I. Ellis, and R. Goodacre, "Metabolomic technologies and their application to the study of plants and plant-host interactions," Physiologia Plantarum, vol. 2, pp. 117-135, 2008.

[22] A. Murai, K. Iwamura, M. Takada, K. Ogawa, T. Usui, and J.-i. Okumura, "Control of postprandial hyperglycaemia by galactosyl maltobionolactone and its novel anti-amylase effect in mice," Life Sciences, vol. 71, no. 12, pp. 1405-1415, 2002.

[23] A. Cencic and W. Chingwaru, "The role of functional foods, nutraceuticals, and food supplements in intestinal health," Nutrients, vol. 2, no. 6, pp. 611-625, 2010.

[24] N. Javadi, F. Abas, A. A. Hamid et al., "GC-MS-based metabolite profiling of Cosmos caudatus leaves possessing alphaglucosidase inhibitory activity," Journal of Food Science, vol. 79, no. 6, pp. 1130-1136, 2014.

[25] L. Liu, M. A. Deseo, C. Morris, K. M. Winter, and D. N. Leach, "Investigation of $\alpha$-glucosidase inhibitory activity of wheat bran and germ," Food Chemistry, vol. 126, no. 2, pp. 553-561, 2011.

[26] S. Murugesu, Z. Ibrahim, Q.-U. Ahmed et al., "Characterization of $\alpha$-glucosidase inhibitors from Clinacanthus nutans lindau leaves by gas chromatography-mass spectrometrybased metabolomics and molecular docking simulation," Molecules, vol. 2402, no. 23, 2018.

[27] B. Na, P.-H. Nguyen, B.-T. Zhao, Q.-H. Vo, B. S. Min, and M. H. Woo, "Protein tyrosine phosphatase 1B (PTP1B) inhibitory activity and glucosidase inhibitory activity of compounds isolated from Agrimonia pilosa," Pharmaceutical Biology, vol. 54, no. 3, pp. 474-480, 2016.

[28] N. Artanti, S. Tachibana, L. B. S. Kardono, and H. Sukiman, "Isolation of $\alpha$-glucosidase Inhibitors Produced by an Endophytic Fungus, Colletotrichum sp. TSC13 from Taxus sumatrana," Pakistan Journal of Biological Sciences, vol. 15, no. 14, p. 673, 2012.

[29] J. N. Kim, S. N. Han, and H. K. Kim, "Phytic acid and myoinositol support adipocyte differentiation and improve insulin sensitivity in 3T3-L1 cells," Nutrition Research, vol. 34, no. 8, pp. 723-731, 2014.

[30] R. Gupta, A. K. Sharma, M. P. Dobhal, M. C. Sharma, and R. S. Gupta, "Antidiabetic and antioxidant potential of $\beta$-sitosterol in streptozotocin-induced experimental hyperglycemia," Journal of Diabetes, vol. 3, no. 1, pp. 29-37, 2011.

[31] A. B. Awad, M. Chinnam, C. S. Fink, and P. G. Bradford, " $\beta$-Sitosterol activates Fas signaling in human breast cancer cells," Phytomedicine, vol. 14, no. 11, pp. 747-754, 2007.

[32] K. Matsuoka, T. Nakazawa, A. Nakamura, C. Honda, K. Endo, and M. Tsukada, "Study of thermodynamic parameters for solubilization of plant sterol and stanol in bile salt micelles," Chemistry and Physics of Lipids, vol. 154, no. 2, pp. 87-93, 2008.

[33] D.-O. Moon, K.-J. Lee, Y. H. Choi, and G.-Y. Kim, " $\beta$-Sitosterol-induced-apoptosis is mediated by the activation of ERK and the downregulation of Akt in MCA-102 murine fibrosarcoma cells," International Immunopharmacology, vol. 7, no. 8, pp. 1044-1053, 2007.
[34] X. Du, Y. Li, Y. L. Xia et al., "Insights into protein-ligand interactions: Mechanisms, models, and methods," International Journal of Molecular Sciences, vol. 17, no. 2, pp. 1-34, 2016.

[35] S. Lu, W. Huang, and J. Zhang, "Recent computational advances in the identification of allosteric sites in proteins," Drug Discovery Today, vol. 19, no. 10, pp. 1595-1600, 2014.

[36] K. Yamamoto, H. Miyake, M. Kusunoki, and S. Osaki, "Crystal structures of isomaltase from Saccharomyces cerevisiae and in complex with its competitive inhibitor maltose," FEBS Journal, vol. 277, no. 20, pp. 4205-4214, 2010.

[37] E. Guarnera and I. N. Berezovsky, "Allosteric sites: remote control in regulation of protein activity," Current Opinion in Structural Biology, vol. 37, pp. 1-8, 2016.

[38] F. Brindis, R. Rodríguez, R. Bye, M. González-Andrade, and R. Mata, "(Z)-3-butylidenephthalide from Ligusticum porteri, an $\alpha$-glucosidase inhibitor," Journal of Natural Products, vol. 74, no. 3, pp. 314-320, 2010.

[39] R. Nussinov, "Introduction to Protein Ensembles and Allostery," Chemical Reviews, vol. 116, no. 11, pp. 6263-6266, 2016.

[40] M. Ali, D. Kim, S. Seong, H.-R. Kim, H. Jung, and J. Choi, " $\alpha$-glucosidase and protein tyrosine phosphatase 1B inhibitory activity of plastoquinones from marine brown alga Sargassum serratifolium," Marine Drugs, vol. 15, no. 12, p. 368, 2017.

[41] X. Zheng, S. Zhou, C. Zhang et al., "Docking-assisted 3DQSAR studies on xanthones as $\alpha$-glucosidase inhibitors," Journal of Molecular Modeling, vol. 23, no. 9, pp. 1-12, 2017. 